\title{
Primary osseous composite sarcoma with focal rhabdomyosarcoma and lymph node metastasis in an adolescent
}

\author{
Maitane Andion ${ }^{1}$, Susana Buendía ${ }^{1}$, Natalia Camarena ${ }^{1}$, Alba Rubio-Sansimón ${ }^{2}$, Teresa \\ Cañas ${ }^{1}$, and Daniel Azorín ${ }^{3}$ \\ ${ }^{1}$ Hospital Niño Jesús. \\ ${ }^{2}$ Hospital Infantil Universitario Niño Jesús \\ ${ }^{3}$ Hospital Infantil Universitario Nino Jesus
}

May 28, 2020

\begin{abstract}
Primary bone composite sarcoma is a very rare entity that primarily affects adolescent and young adult patients. It usually combines areas of liposarcoma and osteosarcoma and up to $60 \%$ of cases have metastatic disease at diagnosis. It is a highly aggressive pathology with intrinsic resistance to conventional treatment schemes for bone sarcomas. The prognosis is poor, with long term survival rates that do not exceed $30 \%$. We present the case of an adolescent female diagnosed with a primary agressive bone composite sarcoma with rhabdomyosarcoma foci and loco-regional lymph node involvement.
\end{abstract}

\section{Introduction:}

Primary bone composite sarcoma is a rare entity that accounts for less than $0.5 \%$ of bone sarcomas. It is diagnosed during adolescence and young adulthood, and usually combines areas of liposarcoma with osteosarcoma. The presence of disseminated pulmonary disease occurs in up to $60 \%$ of patients and, with conventional treatment schemes for osteosarcoma, survival rates do not exceed $30 \%$ (1-5).

We present the case of an adolescent female diagnosed with a primary agressive bone composite sarcoma with rhabdomyosarcoma foci and loco-regional lymph node involvement.

\section{Results:}

A 13-year-old girl without relevant medical antecedents presented with a 4-week history of worsening pain, swelling and loss of strength in her left upper limb. Plain radiography showed an osteolytic lesion involving the proximal humerus, and magnetic resonance imaging (MRI) revealed a bone lesion with characteristics of aggressiveness and associated soft tissue mass (Fig. 1a and b). Trucut biopsy showed a heterogeneous neoplastic proliferation with round cell nests on a collagen stroma and osteoid foci, compatible with conventional osteosarcoma. The bone scan and thoracic computed tomography (CT) ruled out distant metastases but revealed two left axillary and supraclavicular nodular lesions of 17 and $21 \mathrm{~mm}$, respectively (Fig. 1c). An axillary lymph node biopsy was compatible with metastasis of osteosarcoma.

The patient started chemotherapy treatment according to Spanish Society of Pediatric Hematology and Oncology protocol for localized osteosarcoma (SEHOP 2010) (based on methotrexate, cisplatin, doxorubicin, and ifosfamide) presenting a significant decrease in swelling, pain and normalization of analytical parameters in the first weeks of treatment. During induction chemotherapy she suffered from a spontaneous pathological fracture. On week 11 she developed local swelling, pain and increased levels of LDH (lactate dehydrogenase) and alkaline phosphatase. The evaluation carried out on week 12, prior to the loco-regional surgical 
treatment, showed signs of local progression with increase of the soft tissue mass, increased diaphyseal extension and full infiltration of the epiphysis. Local lymph nodes showed radiological stability. The extension work-up ruled out metastatic progression. The patient underwent upper limb amputation and lymph node dissection, since limb sparing surgery was deemed unlikely to achieve optimal local control after discussion in our local multidisciplinary tumour board. The surgical specimen showed a neoplastic proliferation with $30 \%$ post-chemotherapy necrosis and free surgical margins (R0 Ennekin). Atypical cells with epithelioid and focal rhabdoid habit were observed, with positivity for myogenin and desmin. In other areas there were foci of cartilage and fine osteoid (Fig. 2). The neoplasm was reclassified as an osteosarcoma with a focal component of rhabdomyosarcoma. Among the multiple lymph nodes resected, only two of them showed neoplastic infiltration with similar characteristics to those described at bone specimen.

PET-CT performed three weeks after surgery showed new cervical adenopathies, multiple bilateral pulmonary nodules and bone lesions compatible with metastatic progression. She received two cycles of the VIT regimen [vincristine $\left(1.5 \mathrm{mg} / \mathrm{m}^{2} /\right.$ day, days +1 and +8$)$, irinotecan $\left(50 \mathrm{mg} / \mathrm{m}^{2} /\right.$ day x 5 days $)$ and temozolomide (150 mg / $\mathrm{m}^{2} /$ day x 5 days)] in a 21-day interval. She progressed after these two cycles and ultimately died of disease progression.

An extensive molecular analysis was performed on surgical material at progression, including tumour whole exome sequencing (WES), RNA sequencing and methylation profiling. It revealed 21 homologous recombination defects that resulted in a high genomic rearrangement but with a low mutational burden. Mutational disruption of TSC2 (stop-gain mutation), TP53 (stop-gain mutation), CCNE1 (focal duplication and mRNA overexpression) andSETD2 (missense mutation) were revealed. A DNA Methylation-Based Classifier for sarcomas was inconclusive. This analysis was only available after the patient passed away and could not be used for therapeutic purposes.

\section{Discussion:}

The term malignant mesenchymoma was first suggested by Stout in 1948 to define various sarcomas with the common characteristic of being formed by several cell types, excluding fibrosarcoma due to the frequent occurrence of a fibrosarcomatous component associated with most sarcomas (6). Enzinger and Weiss later established the need to clearly differentiate these cell types from a microscopic, immunohistochemical and ultrastructural point of view, also excluding single histologic subtypes as dedifferentiated sarcoma and the combination of osteosarcoma with condrosarcoma (7).

In 1966, Schajowicz described the first case of primary malignant bone mesenchymoma (8), with only about 25 cases reported to date and rarely, cases associated with previous radiation therapy (1-5, 9-18). In recent years this term has been replaced by primary osseous composite sarcoma although this entity is not recognized in the current WHO 2013 classification (5).

Although most malignant soft tissue mesenchymomas are diagnosed at advanced age, primary composite osteosarcoma has a peak incidence in adolescents and young adults, being more frequent in males. The metaphysis of long bones and pelvis are described as the most frequent locations (1-5).

Histologically, these tumors may be derived from a primordial mesenchymal cell with the ability to differentiate into various cell types or they may consist of different cell clones. Previous series have described the combination of areas of liposarcoma with osteosarcoma as the most common pattern (1-5). The present case was diagnosed as an osteosarcoma with rhabdomyosarcoma foci when the entire tumor piece was evaluated. Retrospectively we could demonstrate the focal presence of rhabdoid habit cells with positivity for desmin and myogenin in the diagnostic biopsy that was initially misclassified as conventional osteosarcoma. This histologic variant has been described as the second most common subtype of composite sarcoma, with nine previous cases reported to date. In most of them the diagnosis was not made with the initial biopsy but in the evaluation of the whole tumor specimen, reflecting the diagnostic complexity of these rare forms (1-5, 12-15). The differential diagnosis should incliude dedifferentiated chondrosarcoma, wich is more frequent in older patients, and primary bone rhabdomyosarcoma, another rare entity in children $(1,3,4,13)$. 
Our patient presented with a TSC2 mutation wich is one of the most frequently mutated mTOR pathwayrelated genes. The TSC2 stop-gain mutation likely interferes with the gene function in suppressing the downstream $m T O R$ activity and may lead to more aggressive tumour behaviour (19). It further provides a potential therapeutic role for mTOR inhibitors demonstrated in other solid tumor types that could not be explored in our patient due to the rapid progression (20). Furthermore, SETD2 missense mutation may be an oncogenic driver mutation in this case (21). Its role is known in multiple cancers, including sarcoma, but has not previously been implicated in primary composite osteosarcoma.

From a clinical and radiological point of view, presentation of primary composite bone sarcoma is similar to conventional osteosarcoma, with osteolytic appearance, cortical erosion and periosteal reaction. Nevertheless, the percentage of patients with metastatic disease at diagnosis reaches 50-60\% with pulmonary involvement being the most frequent location (1-5). Our patient presented loco-regional lymph node extension at diagnosis, a rare finding in conventional osteosarcoma related to poor prognosis and not previously described in composite osteosarcoma with rhabdomyosarcoma foci (22-25).

Currently there is not a standar approach on the optimal therapy for primary composite osteosarcoma. It is a highly aggressive pathology with a mortality rate close to $60 \%$, considering that most described cases are treated with chemotherapy schemes for bone or soft tissue sarcoma (1-5).

In conclusion, primary composite osteosarcoma is an exceptional pathology, highly aggressive and intrinsically resistant to chemotherapy, associated with poor prognosis. Tumor sequencing studies may allow the development of targeted treatment strategies in the future. Collaborative efforts are need to gather sufficient biological and clinical information of this condition in children to delineate tailored strategies for them.

Conflicts of interest: none to declare.

The manuscript has not been submitted elsewhere nor previously published.

All authors have contributed to the manuscript in significant ways, have reviewed and agreed upon the manuscript content.

Acknowledgements: none.

References

1. Kessler S, Mirra JM, Ishii T, Thompson JC, Brien EW. Primary malignant mesenchymoma of bone: case report, literature review, and distinction of this entity from mesenchymal and dedifferentiated chondrosarcoma. Skeletal Radiol 1995 May;24(4):291-5.

2. Van Dorpe J, Sciot R, Samson I, De Vos R, Brys P, Van Damme B. Primary osteorhabdomyosarcoma (malignant mesenchymoma) of bone: a case report and review of the literature. Mod Pathol 1997 Oct;10(10):1047-53.

3. Chow LT, Kumta SM. Primary osteochondrorhabdomyosarcoma (malignant mesenchymoma) of the fibula: a rare tumour in an unusual site - case report and review of the literature. APMIS 2004 Sep;112(9):617-23.

4. Mrad K, Sassi S, Smida M, Oubiche F, Mekni A, Romdhane KB. Osteosarcoma with rhabdomyosarcomatous component or so-called malignant mesenchymoma of bone. Pathologica2004 Dec;96(6):475-8.

5. Chow LT. Critical reappraisal of primary osseous composite sarcoma (malignant mesenchymoma) - analysis of four cases and literature review. APMIS 2016 Jun;124(6):487-99. doi: 10.1111/apm.12540. Epub 2016 Apr 22.

6. Stout AP. Mesenchymoma: the mixed tumour of mesenchymal derivates . Ann Surg 1948;127:278-290. 
7. Enzinger RM, Weiss SW. Malignant soft tissue tumors of uncertain type. In: Soft Tissue Tumors, $3^{\text {rd }}$ Ed. St Louis: Mosby-Year Book, Inc., 1995:1067-93 (1087-89).

8. Schajowicz F, Cuevillas R, Silberman FS. Primary malignant mesenchymoma of bone. A new tumor entity. Cancer 1966 Oct;19(10):1423-8.

9. Small GS. Malignant mesenchymoma of the mandible . Oral Surg Oral Med Pathol 1961 Dec;14:1427-35.

10. Ross CF, Hadfield G. Primary osteoliposarcoma of bone (malignant mesenchymoma). Report of a case. J Bone Joint Surg Br 1968 Aug;50(3):639-43.

11. Sterns EE, Haust MD, Wollin DG. Malignant mesenchymoma of the mandible . Can J Surg 1969 Oct;12(4):444-9.

12. Scheele PM, Von Kuster LC, Krivchenia G. Primary malignant mesenchymoma of bone . Arch Pathol Lab Med 1990 Jun;114(6):614-7.

13. Marcial-Seoane RA, Marcial-Seoane MA, Dávila-Toro FL, Marcial-Rojas RA. Bone tumors of mixed origin: osteoliposarcoma and osteorhabdomyosarcoma. Bol Asoc Med P R 1990 Sep;82(9):378-93.

14. Jain SK, Afzal M, Mathew M, Ramani SK. Malignant mesenchymoma of the chest wall in an adult. Thorax 1993 Apr;48(4):407-8.

15. Lamovec J, Zidar A, Bracko M, Golouh R. Primary bone sarcoma with rhabdomyosarcomatous component. Pathol Res Pract 1994 Jan;190(1):51-60.

16. De Padua M, Bhandari TP, Pingle J.Primary osteoliposarcoma of the bone. Indian J Pathol Microbiol 2009 Jan-Mar;52(1):80-2.

17. Sade R, Yuce I, Karaca L, Ogul H, Kantarci M, Levent A. Malignant mesenchymal tumor of the sacrum.Spine J 2015 Oct 1;15(10):e39-40.

18. Kudawara I, Araki N, Nakanishi H, Mano M, Ishiguro S. Malignant mesenchymoma of the lower leg. J Clin Pathol 2001 Nov;54(11):877-9.

19. Pan CC, Chung MY, Ng KF, Liu CY, Wang JS, Chai CY, et al.Constant allelic alteration on chromosome 16p (TSC2 gene) in perivascular epithelioid cell tumour (PEComa): genetic evidence for the relationship of PEComa with angiomyolipoma. Pathol 2008 Feb;214(3):387-93.

20. Lim SM, Park HS, Kim S, Kim S, Ali SM, Greenbowe JR, et al.Next-generation sequencing reveals somatic mutations that confer exceptional response to everolimus. Oncotarget 2016 Mar $1 ; 7(9): 10547-56$.

21. Tokheim C, Karchin R. CHASMplus reveals the scope of somatic missense mutations driving human cancers. Cell Syst 2019 Jul 24;9(1):9-23.

22. Thampi S, Matthay KK, Goldsby R, DuBois SG. Adverse impact of regional lymph node involvement in osteosarcoma. Eur J Cancer 2013 Nov;49(16):3471-6.

23. Fioravantti V, Sirvent S, De Prada I, Bautista F.Radiological regional lymph node involvement with no histopathology correlation in an osteosarcoma of the humerus . Ann Pediatr (Barc) 2017 Oct;87(4):237-239.

24. Hattori H, Yamamoto K. Lymph node metastasis of osteosarcoma . J Clin Oncol 2012 Nov 20;30(33):e345-9.

25. Oñoro G, Hernández C, Sirvent S, Aleo E, Molina B, Lassaletta a, et al. Unusual sites of extrapulmonary metastases of osteosarcoma after several lines of treatment. Pediatr Hematol Oncol 2011 Oct;28(7):604-8. 


\section{Legends:}

Fig 1.

Coronal plane T1 MRI images (a) and STIR (b) in which there is a bone marrow signal replacement of the proximal humerus, which is hypointense in T1 and hyperintense in STIR, associated whith a soft tissues mass sorrounding the humerus circumferentially.

Coronal chest CT scan (c) showing two left axillary/infraclavicular adenopathies of suspicious appearance (arrow).

\section{Fig. 2.}

Core biopsy showing an osteogenic malignant tumor with permeative growth pattern. Notice the formation of osteoid at the left side and an epithelioid/rhabdoid cell proliferation at the right side (a). Spotted cells stained with desmin (b) and myogenin (c).
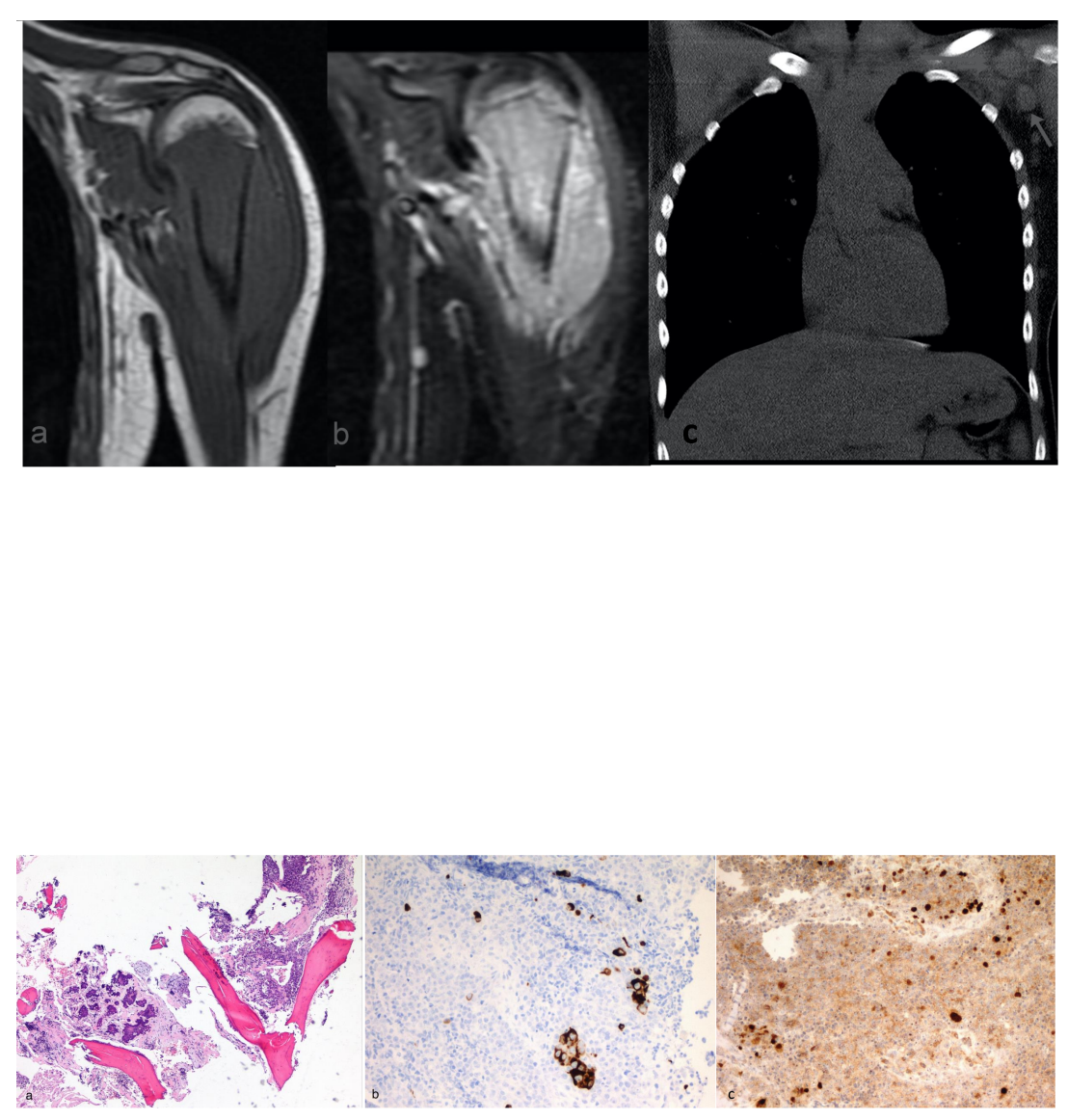